\title{
Calidad de vida de los pacientes en diálisis. Revisión sistemática
}

\author{
Azahara $\mathbf{M}^{\mathrm{a}}$ Sánchez-Cabezas ${ }^{1}$, Noelia Morillo-Gallego ${ }^{1}$, Rosa $^{\mathrm{a}}{ }^{\mathrm{a}}$ Merino-Martínez ${ }^{1}$, Rodolfo Crespo-Montero ${ }^{1,2,3}$ \\ ${ }^{1}$ Departamento de Enfermería. Facultad de Medicina y Enfermería. Universidad de Córdoba. España \\ ${ }^{2}$ Servicio de Nefrología. Hospital Universitario Reina Sofía de Córdoba. España \\ ${ }^{3}$ Instituto Maimónides de Investigación Biomédica de Córdoba. España
}

Como citar este artículo:

Sánchez-Cabezas AM, Morillo-Gallego N, Merino-Martínez RM, Crespo-Montero R. Calidad de vida de los pacientes en diálisis. Revisión sistemática. Enferm Nefrol. 2019 Jul-Sep;22(3):239-55

\section{Resumen}

Objetivo: Identificar las variables más influyentes en la calidad de vida relacionada con la salud en pacientes en diálisis, identificando los instrumentos de medida más utilizados.

Material y Método: Se ha realizado una revisión sistemática en las bases de datos PubMed, Scopus, Google Académico y Scielo. Se han incluido artículos científicos en inglés y español. Se han analizado artículos que tratan la calidad de vida relacionada con la salud en pacientes en diálisis, excluyendo aquellos artículos que no aportaban resultados concluyentes, en población pediátrica y en los que se analizaba este parámetro exclusivamente en pacientes trasplantados.

Resultados: Se han incluido 36 artículos publicados entre 2009-2019. La calidad de vida relacionada con la salud aparece disminuida en todos los pacientes en diálisis, más concretamente en hemodiálisis. Las variables más influyentes en la disminución de la calidad de vida relacionada con la salud del paciente en diálisis, son: sexo femenino, edad avanzada, no poseer un núcleo familiar, estudios primarios, y afectación de la situación laboral en pacientes en edad para trabajar; además de comorbilidad asociada a la patología renal, ansiedad y depresión, presencia de dolor y con grado de dependencia alto.

Conclusiones: La disminución de la calidad de vida está presente en la mayoría de los pacientes de diáli-

\section{Correspondencia: \\ Azahara M ${ }^{a}$ Sánchez Cabezas \\ C/ Luna 7. 14500 Puente Genil. Córdoba \\ E-mail: azaharasc93@hotmail.com}

sis, con más frecuencia en hemodiálisis. Respecto a los instrumentos de evaluación empleados, el más utilizado es el KDQOL-SF. Esta pérdida de la calidad de vida se relaciona con ser mujer, edad avanzada, comorbilidad asociada, dolor con limitaciones, presencia de ansiedad y/o depresión y mayor dependencia.

PALABRAS CLAVE: diálisis; calidad de vida relacionada con la salud; hemodiálisis; diálisis peritoneal.

\section{Quality of life of dialysis patients. Systematic review}

\section{Abstract}

Objective: To identify the most influential variables in health-related quality of life in dialysis patients, identifying the most common measuring instruments.

Material and Method: A systematic review was carried out in the PubMed, Scopus, Google Scholar and Scielo databases. Scientific articles in English and Spanish have been included. Articles that evaluate health-related quality of life in dialysis patients, excluding those articles that did not provide conclusive results, in the pediatric population and those that addressed this parameter exclusively in transplanted patients, were analyzed.

Results: 36 articles published between 2009-2019 have been included. Health-related quality of life appears diminished in all dialysis patients, more specifically in hemodialysis. The most influential variables in the decrease of the health-related quality of life of the dialysis patient are: female sex, older age, not having a family nucleus, primary studies, and affectation of the employment situation in patients of working age; in addition to comorbidity 
associated with kidney disease, anxiety and depression, presence of pain and high degree of dependence.

Conclusions: The decrease in the quality of life is present in many dialysis patients, more frequently in hemodialysis. Regarding the evaluation instruments used, the most used is the KDQOL-SF. This loss of quality of life is related to being a woman, old age, associated comorbidity, pain with limitations, presence of anxiety and/or depression and greater dependence.

KEYWORDS: dialysis; health-related quality of life; hemodialysis; peritoneal dialysis.

\section{Introducción}

La enfermedad renal crónica es una alteración que se presenta de forma silenciosa y ha pasado a constituir un problema de Salud Pública en todo el mundo, debido al aumento de los casos diagnosticados en los últimos años. En España, se estima que la enfermedad renal crónica es más frecuente en mayores de 60 años y género masculi$n 0^{1,2}$.

La Enfermedad Renal Crónica Avanzada (ERCA), además de las repercusiones clínicas en todos los sistemas y aparatos del organismo, va a originar cambios importantes en la calidad de vida de los pacientes a raíz del transcurso y evolución de la enfermedad, influyendo en esto factores sociodemográficos, comorbilidad asociada, complicaciones de la propia enfermedad o factores de riesgo, y los tratamientos disponibles, provocando un deterioro tanto a nivel físico como psicológico en dicha calidad de vida de los paciente ${ }^{3,4}$.

La Organización Mundial de la Salud, definió Calidad de Vida como "la percepción del individuo de su posición en la vida en el contexto de la cultura y el sistema de valores en los que vive y en relación a sus metas, expectativas, valores y preocupaciones ${ }^{\prime \prime 5}$. Sin embargo, en las alteraciones crónicas, como la ERCA, el concepto más idóneo a evaluar en este terreno, sería la Calidad de Vida Relacionada con la Salud (CVRS), que según definen Shumaker y Naughton, hace referencia a "la evaluación subjetiva de las influencias del estado de salud actual, los cuidados sanitarios, y la promoción de la salud sobre la capacidad del individuo para lograr y mantener un nivel global de funcionamiento que permite seguir aquellas actividades que son importantes para el individuo y que afectan a su estado general de bienestar"6. Este es un concepto multidimensional y complejo, que incluye aspectos personales como salud, satisfacción con la vida y autonomía y aspectos ambientales como grupos sociales y de apoyo, entre otros ${ }^{7}$, y se empieza a tomar conciencia de ello debido al aumento de las enfermedades crónicas en la población, ya que la persona que padece este tipo de enfermedades no solo tiene que sobrellevar la patología como tal, sino que además, tiene que luchar contra los efectos adversos de los tratamientos que le son administrados ${ }^{8}$.

El concepto de CVRS se compone de diferentes situaciones en las que el paciente ve alteradas ciertas dimensiones corporales, como funcionamiento físico, bienestar psicológico, estado emocional, dolor, funcionamiento social, percepción general de la salud, el grado de satisfacción con la vida, el impacto sobre la productividad laboral y las actividades de la vida diaria?

La importancia de medir la CVRS radica en la estrecha relación que existe entre CVRS, morbilidad y mortalidad, en los pacientes con ERCA en diálisis, por lo que es importante saber en todo momento, cómo el paciente percibe su estado de salud durante los tratamientos de sustitución renal, al mismo tiempo que ayuda a detectar algún cambio que se esté produciendo y que no esté siendo percibido por el paciente ${ }^{4,10}$.

Debido al carácter subjetivo que tiene medir calidad de vida, surgen instrumentos de medida validados para la CVRS que son utilizados con el fin de obtener un resultado objetivo y comparable de este concepto. Estos instrumentos pueden ser genéricos o específicos, compuestos por diferentes ítems o preguntas que miden distintos aspectos de la salud. El más utilizado es de tipo genérico, el SF-36, y ofrece una idea de cómo el paciente percibe su estado de salud, a través de 36 ítems organizados en 8 dimensiones ${ }^{11}$.

La medida de la CVRS permite realizar una evaluación continua del paciente desde el diagnóstico de la enfermedad, hasta las etapas más avanzadas de la enfermedad, haciendo posible planificar unos cuidados personalizados y centrados en aquellas dimensiones que se encuentran alteradas en ese momento ${ }^{12}$.

Por todo ello, dada la importancia que tiene la percepción de la CVRS en el paciente en HD o DP, es por lo que hemos decidido llevar a cabo esta revisión, con el propósito de conocer cuáles son las variables más influyentes en la calidad de vida de estos pacientes. 
Por tanto, el propósito de esta revisión ha sido identificar las variables más influyentes en la CVRS en pacientes en diálisis, identificando los instrumentos de medida más utilizados.

\section{Metodología}

\section{Diseño y ámbito de estudio}

Se ha llevado a cabo una revisión bibliográfica sistematizada.

El ámbito de estudio han sido las bases de datos: PubMed, Scopus, Google Académico y Scielo.

\section{Objeto de estudio}

Documentos encontrados en las bases de datos seleccionadas.

\section{Criterios de inclusión:}

- Artículos originales, artículos de revisión, artículos en inglés y en español y estudio en humanos.

- Estudios en los cuales se tratara la calidad de vida en de los pacientes de diálisis.

\section{Criterios de exclusión:}

- Artículos sin texto completo disponible o que no presentaban resultados.

- Artículos cuya población de estudio fueran pacientes pediátricos.

\section{Variables}

La búsqueda en bases de datos con motores de búsqueda en inglés se llevó a cabo utilizando las palabras clave: quality of life, quality of life related of health, chronic kidney disease, dialysis, hemodialysis, peritoneal dialysis.

\section{Periodo de estudio}

En el estudio se incluyeron aquellos documentos publicados entre los años 2009 y 2018.

\section{Periodo de recogida de datos}

La búsqueda ha sido realizada durante el periodo comprendido entre octubre de 2018 y febrero de 2019.

Procedimiento, recogida de datos y diagrama de flujo Para realizar la revisión sistemática se siguieron las recomendaciones de la revisión PRISMA ${ }^{13}$, cuya creación ha surgido con el fin de mejorar la claridad, la transparencia y el desarrollo homogéneo de las revisiones sistemáticas. Para la estrategia de búsqueda se utilizaron diferentes combinaciones entre las variables y los operadores Booleanos "AND" y "OR".

La selección de artículos se realizó mediante la lectura del título y/o resumen y posteriormente, mediante la aplicación de los criterios de inclusión y exclusión.

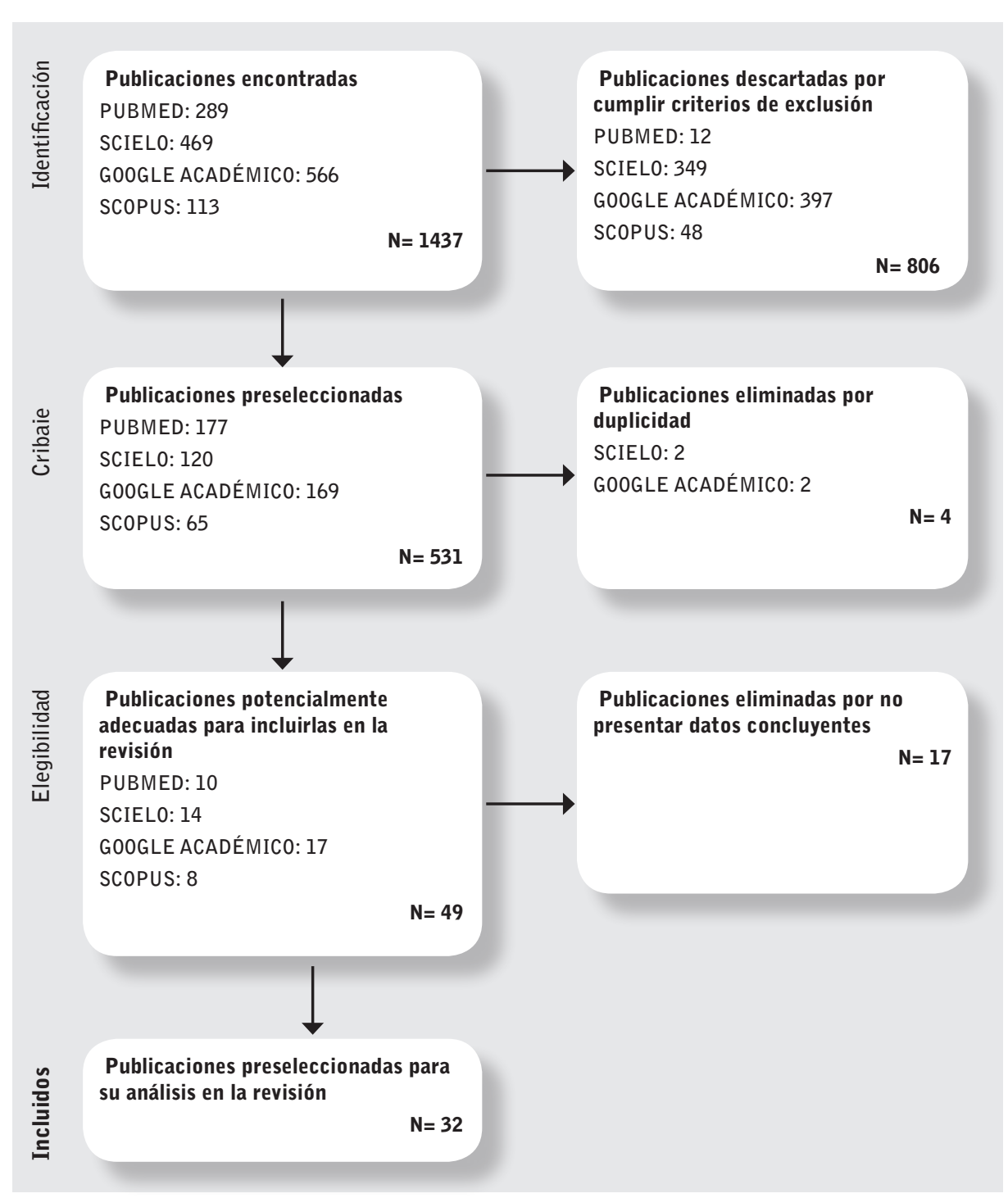

Figura 1. Diagrama de flujo de la estrategia de búsqueda. 
Se revisaron los títulos y resúmenes de 49 artículos, de los cuales 36 fueron revisados a texto completo. Posteriormente, 13 fueron excluidos por no cumplir los criterios de inclusión propuestos y no dar resultados claros.

Los detalles del proceso de búsqueda se muestran en el diagrama de flujo (Figura 1).

\section{Análisis de los datos}

Con el fin de evaluar la calidad metodológica de los artículos se utilizó la lista de verificación STROBE ${ }^{14}$ para los estudios observacionales y transversales (Tabla $\mathbf{1}$ ).

Tabla 1. Descripción de las características de los artículos seleccionados.

\begin{tabular}{|c|c|c|}
\hline TÍTULO & Tipo de estudio & STROBE (0-22) \\
\hline Pérez Acuña $C$ et al ${ }^{15}$ & Transversal & 19 \\
\hline Seguí Gomá $A$ et al ${ }^{18}$ & Descriptivo de corte transversal & 20 \\
\hline Zúñiga SM C et al ${ }^{20}$ & Transversal & 19 \\
\hline Perales Montilla CM et a ${ }^{21}$ & Transversal & 20 \\
\hline Varela $L$ et $\mathrm{al}^{22}$ & Transversal & 21 \\
\hline Cuevas Budhart MA et $\mathrm{a}^{23}$ & Transversal analítico & 21 \\
\hline Ho SE et $\mathrm{al}^{24}$ & Transversal descriptivo & 20 \\
\hline Barros Higgins L et al $\left.\right|^{25}$ & Transversal & 20 \\
\hline Millán González R et al²6 & Transversal & 20 \\
\hline Adell Lleixá $\mathrm{M}$ et $\mathrm{al}^{27}$ & Transversal & 17 \\
\hline López y López LR et al ${ }^{28}$ & Transversal & 21 \\
\hline Hernández Mariano JA et al ${ }^{29}$ & Transversal & 20 \\
\hline Albañil Frías $T$ et al ${ }^{30}$ & Observacional analítico de corte transversal & 20 \\
\hline Seok Hui Kang et al ${ }^{31}$ & Transversal & 19 \\
\hline Iyasere $0 \mathrm{U}$ et $\mathrm{al}^{32}$ & Observacional comparativo de corte transversal & 22 \\
\hline Liebman $\mathrm{S}$ et a ${ }^{33}$ & Transversal & 20 \\
\hline Carpena Ramos EC et al ${ }^{34}$ & Transversal & 20 \\
\hline Loon et al ${ }^{36}$ & Aleatorio multicéntrico & 21 \\
\hline García Llana $\mathrm{H}$ et al ${ }^{37}$ & Autoinformes & 19 \\
\hline Barbero Narbona $E$ et a $\left.\right|^{38}$ & Descriptivo de corte transversal prospectivo & 21 \\
\hline Besey Oren et a ${ }^{39}$ & Transversal & 19 \\
\hline Ruiz de alegría $B$ et al ${ }^{40}$ & Longitudinal transversal & 21 \\
\hline Nagasawa $\mathrm{H}$ et al ${ }^{41}$ & Transversal & 19 \\
\hline Atapour A et al ${ }^{42}$ & Transversal & 20 \\
\hline Perales Montilla CM et $\mathrm{al}^{43}$ & Comparativo de corte transversal & 21 \\
\hline González FA et al ${ }^{44}$ & Transversal & 19 \\
\hline Gunalay $\mathrm{S}$ et al ${ }^{45}$ & Transversal & 20 \\
\hline Karatas A et al ${ }^{46}$ & Transversal & 18 \\
\hline
\end{tabular}




\section{Resultados}

\section{Características de los estudios}

Los datos más relevantes de los estudios se muestran en la Tabla 2.

Tabla 2. Características de los documentos incluidos en la revisión.

\begin{tabular}{|c|c|c|c|c|c|}
\hline $\begin{array}{l}\text { Autor, año y } \\
\text { país }\end{array}$ & $\begin{array}{l}\text { Diseño del } \\
\text { estudio }\end{array}$ & Muestra & $\begin{array}{l}\text { Instrumento de } \\
\text { medida }\end{array}$ & Objetivo & Conclusiones \\
\hline $\begin{array}{l}\text { Pérez Acuña C } \\
\text { et al. } 2015^{15} \\
\text { Chile } \\
\text { SJR: Q3 }\end{array}$ & $\begin{array}{l}\text { Observacional } \\
\text { de corte } \\
\text { transversal }\end{array}$ & $100 *$ & $\begin{array}{l}\text { IPQ-R } \\
\text { KDQOL-SF }\end{array}$ & $\begin{array}{l}\text { Evaluar la } \\
\text { asociación } \\
\text { entre la ERCA } \\
\text { y percepción de } \\
\text { CVRS en HD. }\end{array}$ & $\begin{array}{l}\text { A mayor comprensión de la } \\
\text { enfermedad menor representación } \\
\text { emocional y mayor CVRS, por ello } \\
\text { el equipo de salud debe dirigir } \\
\text { las intervenciones al proceso de } \\
\text { comprensión de la enfermedad, para } \\
\text { mejorar la percepción de CVRS. }\end{array}$ \\
\hline $\begin{array}{l}\text { Rebollo Rubio } \\
\text { A et al. } 2015^{16} \\
\text { España } \\
\text { SJR: Q3 }\end{array}$ & $\begin{array}{l}\text { Revisión } \\
\text { Sistemática }\end{array}$ & $53 * *$ & $\begin{array}{l}\text { SF-36 } \\
\text { KDQOL-SF } \\
\text { Sickness Impact } \\
\text { Profile } \\
\text { COOP-WONCA }\end{array}$ & $\begin{array}{l}\text { Ofrecer una } \\
\text { comparación de } \\
\text { los instrumentos } \\
\text { de evaluación } \\
\text { de la CVRS } \\
\text { más usados } \\
\text { en población } \\
\text { española con } \\
\text { ERCA. }\end{array}$ & $\begin{array}{l}\text { Los estudios en su mayoría muestran } \\
\text { cómo la CVRS se ve afectada de } \\
\text { forma importante en pacientes } \\
\text { con tratamiento renal sustitutivo, } \\
\text { independientemente del instrumento } \\
\text { usado para medir la CVRS. Se } \\
\text { necesitan más estudios que aborden } \\
\text { este parámetro para concretar datos, } \\
\text { así como estudios cuyas muestran sean } \\
\text { más grandes. }\end{array}$ \\
\hline $\begin{array}{l}\text { Zazzeroni L et } \\
\text { al. } 2017^{17} \\
\text { Italia } \\
\text { SJR: Q } 2\end{array}$ & $\begin{array}{l}\text { Revisión } \\
\text { sistemática y } \\
\text { metanálisis }\end{array}$ & $7 * *$ & KDQOL-SF 1.3036 & $\begin{array}{l}\text { Determinar qué } \\
\text { tratamiento, HD } \\
\text { o DP, proporciona } \\
\text { mejor CVRS. }\end{array}$ & $\begin{array}{l}\text { El análisis no conduce a una } \\
\text { conclusión unánime. Se muestra que } \\
\text { estadísticamente la única diferencia } \\
\text { significativa entre la CVRS de los } \\
\text { pacientes con HD y DP se refiere al } \\
\text { efecto de la enfermedad renal, siendo } \\
\text { mejor en DP. }\end{array}$ \\
\hline $\begin{array}{l}\text { Seguí Gomá A } \\
\text { et al. } 2010^{18} \\
\text { España } \\
\text { SJR: Q3 }\end{array}$ & $\begin{array}{l}\text { Observacional } \\
\text { descriptivo } \\
\text { de corte } \\
\text { transversal }\end{array}$ & $96 *$ & SF-36 & $\begin{array}{l}\text { Evaluar la CVRS } \\
\text { y características } \\
\text { personales de } \\
\text { pacientes con IRC } \\
\text { en HD. }\end{array}$ & $\begin{array}{l}\text { La calidad de vida en pacientes con } \\
\text { IRCT en HD, es peor que la población } \\
\text { de referencia en todas las dimensiones } \\
\text { analizadas. }\end{array}$ \\
\hline $\begin{array}{l}\text { Boateng EA et } \\
\text { al. } 2011^{19} \\
\text { Ghana } \\
\text { SJR: Q2 }\end{array}$ & $\begin{array}{l}\text { Revisión } \\
\text { sistemática }\end{array}$ & 26 ** & $\begin{array}{l}\text { SF-36 } \\
\text { KDQOL } \\
\text { CHEQ }\end{array}$ & $\begin{array}{l}\text { Generar evidencia } \\
\text { sobre qué diálisis } \\
\text { (HD/DP) mejora } \\
\text { la calidad de vida } \\
\text { de los pacientes } \\
\text { con ERCA }\end{array}$ & $\begin{array}{l}\text { No existe una respuesta clara sobre } \\
\text { la modalidad que mejora la CVRS Sin } \\
\text { embargo, una buena comprensión de } \\
\text { la base de evidencia facilitará la toma } \\
\text { de decisiones individuales. }\end{array}$ \\
\hline $\begin{array}{l}\text { Zúñiga SM C } \\
\text { et al. } 2009^{20} \\
\text { Chile } \\
\text { SJR: Q3 }\end{array}$ & $\begin{array}{l}\text { Estudio } \\
\text { multicéntrico } \\
\text { de corte } \\
\text { transversal }\end{array}$ & $224^{*}$ & $\begin{array}{l}\text { KDQOL-36 } \\
\text { ESCALA DE } \\
\text { KARNOSFSKY }\end{array}$ & $\begin{array}{l}\text { Evaluar la CVRS } \\
\text { en un grupo de } \\
\text { pacientes con HD } \\
\text { crónica. }\end{array}$ & $\begin{array}{l}\text { La CVRS en pacientes en HD tuvo } \\
\text { valores inferiores a los de referencia } \\
\text { en sujetos con diabetes mellitus y } \\
\text { enfermedad coronaria, mal estado } \\
\text { nutricional y bajo nivel educativo y } \\
\text { socioeconómico. }\end{array}$ \\
\hline
\end{tabular}




\begin{tabular}{|c|c|c|c|c|c|}
\hline $\begin{array}{l}\text { Autor, año y } \\
\text { país }\end{array}$ & $\begin{array}{l}\text { Diseño del } \\
\text { estudio }\end{array}$ & Muestra & $\begin{array}{l}\text { Instrumento de } \\
\text { medida }\end{array}$ & Objetivo & Conclusiones \\
\hline $\begin{array}{l}\text { Perales } \\
\text { Montilla CM } \\
\text { et al. } 2012 \\
\text { España } \\
\text { SJR: Q3 }\end{array}$ & $\begin{array}{l}\text { Estudio } \\
\text { transversal }\end{array}$ & $39 *$ & SF-36 & $\begin{array}{l}\text { Valorar CVRS } \\
\text { de los pacientes } \\
\text { con ERCA en } \\
\text { HD, y evaluar } \\
\text { la capacidad } \\
\text { predictiva de } \\
\text { algunas variables } \\
\text { psicosociales } \\
\text { sobre la CVRS. }\end{array}$ & $\begin{array}{l}\text { Los resultados del estudio sugieren } \\
\text { que la intervención en aspectos } \\
\text { psicosociales puede resultar de gran } \\
\text { interés para mejorar la CVRS, ya que } \\
\text { el principal predictor de CVRS es la } \\
\text { depresión. Esto desencadenará en el } \\
\text { paciente una mejor adaptación a la } \\
\text { enfermedad, mejorando el pronóstico } \\
\text { y la respuesta al tratamiento en los } \\
\text { pacientes en HD. }\end{array}$ \\
\hline $\begin{array}{l}\text { Varela L et al. } \\
2011^{22} \\
\text { España } \\
\text { SJR: ND }\end{array}$ & $\begin{array}{l}\text { Estudio } \\
\text { observacional } \\
\text { transversal }\end{array}$ & $53^{*}$ & $\begin{array}{l}\text { Kidney Disease } \\
\text { Quality of Life Short } \\
\text { Form }\end{array}$ & $\begin{array}{l}\text { Identificar los } \\
\text { predictores } \\
\text { psicológicos } \\
\text { de la CVRS en } \\
\text { pacientes en DP. }\end{array}$ & $\begin{array}{l}\text { En los pacientes con DP, la ansiedad, } \\
\text { la depresión y la alexitimia son } \\
\text { importantes determinantes de } \\
\text { la CVRS, por lo que deberían } \\
\text { ser incluidos en la evaluación y } \\
\text { tratamiento de esta población de } \\
\text { enfermos. }\end{array}$ \\
\hline $\begin{array}{l}\text { Cuevas } \\
\text { Budhart M.A. } \\
\text { et al. } 2017^{23} \\
\text { México } \\
\text { SJR: Q3 }\end{array}$ & $\begin{array}{l}\text { Transversal } \\
\text { analítico }\end{array}$ & $157^{*}$ & KDQ0L-36 & $\begin{array}{l}\text { Evaluar la CVRS } \\
\text { de pacientes en } \\
\text { HD y determinar } \\
\text { la asociación } \\
\text { entre las } \\
\text { complicaciones y } \\
\text { la CVRS. }\end{array}$ & $\begin{array}{l}\text { Las complicaciones del tratamiento } \\
\text { de HD aunadas a las de la ERC } \\
\text { deterioran en gran medida la calidad } \\
\text { de vida del paciente. }\end{array}$ \\
\hline $\begin{array}{l}\text { Ho SE et al. } \\
2013^{24} \\
\text { Malasia } \\
\text { SJR: Q3 }\end{array}$ & $\begin{array}{l}\text { Estudio } \\
\text { descriptivo } \\
\text { transversal }\end{array}$ & $72^{*}$ & KDQOL-SF & $\begin{array}{l}\text { Examinar la } \\
\text { CVRS entre los } \\
\text { pacientes en } \\
\text { HD de ERCA en } \\
\text { Malasia. }\end{array}$ & $\begin{array}{l}\text { Los pacientes sometidos a HD tienen } \\
\text { buena CVRS a pesar de tener ERCA. } \\
\text { Por lo tanto, es de gran importancia } \\
\text { monitorear constantemente el nivel } \\
\text { de atención para estos pacientes para } \\
\text { que puedan vivir su vida al máximo. }\end{array}$ \\
\hline $\begin{array}{l}\text { Barros-Higgins } \\
\text { L et al. } 2015^{25} \\
\text { Colombia } \\
\text { SJR: Q4 }\end{array}$ & $\begin{array}{l}\text { Estudio } \\
\text { analítico } \\
\text { de corte } \\
\text { transversal } \\
\text { prospectivo un } \\
\text { año atrás }\end{array}$ & $80^{*}$ & $\begin{array}{l}\text { KDQ0L-SF } \\
\text { Test psicológicos }\end{array}$ & $\begin{array}{l}\text { Determinar la } \\
\text { CVRS de los } \\
\text { pacientes con } \\
\text { ERC y su relación } \\
\text { con variables } \\
\text { sociodemográficas } \\
\text { y datos clínicos en } \\
\text { pacientes de HD. }\end{array}$ & $\begin{array}{l}\text { La ERCA tiene una carga sobre la } \\
\text { CVRS de los pacientes en términos de } \\
\text { salud física, salud mental y carga de la } \\
\text { enfermedad. }\end{array}$ \\
\hline $\begin{array}{l}\text { Millán } \\
\text { González R et } \\
\text { al. } 2009^{26} \\
\text { Colombia } \\
\text { SJR: Q4 }\end{array}$ & $\begin{array}{l}\text { Estudio } \\
\text { multicéntrico } \\
\text { descriptivo, } \\
\text { de corte } \\
\text { transversal }\end{array}$ & $174^{*}$ & $\begin{array}{l}\text { SF-36 } \\
\text { HADS } \\
\text { Fichas con datos } \\
\text { sociodemográficos }\end{array}$ & $\begin{array}{l}\text { Determinar } \\
\text { la CVRS y la } \\
\text { prevalencia } \\
\text { de depresión y } \\
\text { ansiedad. }\end{array}$ & $\begin{array}{l}\text { La CVRS es comparable con la } \\
\text { muestra hallada en otras poblaciones, } \\
\text { excepto por el rango anormalmente } \\
\text { bajo de desempeño físico. }\end{array}$ \\
\hline $\begin{array}{l}\text { Adell Lleixá M } \\
\text { et al. } 2015^{27} \\
\text { España } \\
\text { SJR: Q3 }\end{array}$ & $\begin{array}{l}\text { Estudio } \\
\text { observacional, } \\
\text { analítico } \\
\text { de corte } \\
\text { transversal }\end{array}$ & $44^{*}$ & $\begin{array}{l}\text { KDQL-SF } \\
\text { Datos clínicos y socio- } \\
\text { demográficos }\end{array}$ & $\begin{array}{l}\text { Evaluar las } \\
\text { variables } \\
\text { sociodemográficas } \\
\text { y los parámetros } \\
\text { analíticos de las } \\
\text { personas con } \\
\text { ERCT en HD } \\
\text { y estudiar la } \\
\text { relación con la } \\
\text { CVRS. }\end{array}$ & $\begin{array}{l}\text { Vivir solo y tener estudios se relaciona } \\
\text { con mejores puntuaciones en el test } \\
\text { de CVRS. Las personas que reciben } \\
\text { tratamiento con HDF-OL presentan } \\
\text { mejor estado nutricional que las que } \\
\text { reciben tratamiento con HD-AF, al } \\
\text { igual que mejor puntuación en el } \\
\text { test KDQOLSF. Aunque las mujeres } \\
\text { se dializan mejor que los hombres y } \\
\text { presentan mejores puntaciones en las } \\
\text { escalas de función sexual y situación } \\
\text { laboral, estas manifiestan tener más } \\
\text { dolor, peor estado físico y menor } \\
\text { vitalidad. }\end{array}$ \\
\hline
\end{tabular}




\begin{tabular}{|c|c|c|c|c|c|}
\hline $\begin{array}{l}\text { Autor, año y } \\
\text { país }\end{array}$ & $\begin{array}{l}\text { Diseño del } \\
\text { estudio }\end{array}$ & Muestra & $\begin{array}{l}\text { Instrumento de } \\
\text { medida }\end{array}$ & Objetivo & Conclusiones \\
\hline $\begin{array}{l}\text { López y López } \\
\text { LR et al. } \\
2017^{28} \\
\text { México } \\
\text { SJR: Q4 }\end{array}$ & $\begin{array}{l}\text { Estudio } \\
\text { observacional, } \\
\text { transversal, } \\
\text { descriptivo y } \\
\text { multicéntrico }\end{array}$ & $42^{*}$ & KDQ0L-SF 36 & $\begin{array}{l}\text { Demostrar mayor } \\
\text { puntuación en la } \\
\text { escala de CVRS } \\
\text { en pacientes con } \\
\text { DP comparados } \\
\text { con pacientes en } \\
\text { HD. }\end{array}$ & $\begin{array}{l}\text { En pacientes con diálisis peritoneal el } \\
\text { puntaje es mejor que en pacientes en } \\
\text { hemodiálisis en cuanto a efectos de la } \\
\text { enfermedad renal. También se observó } \\
\text { menor puntaje en pacientes en diálisis } \\
\text { peritoneal vs hemodiálisis en carga de } \\
\text { la enfermedad renal, área de efectos } \\
\text { mentales, efectos físicos y área de } \\
\text { síntomas, sin diferencia estadística. } \\
\text { Tampoco hubo diferencia significativa } \\
\text { en diálisis a largo plazo (más de } \\
\text { cuatro años) entre ambos grupos. Por } \\
\text { tanto, el médico y el paciente deben } \\
\text { encontrar la modalidad de diálisis que } \\
\text { se ajuste mejor a sus necesidades y } \\
\text { estilo de vida. }\end{array}$ \\
\hline $\begin{array}{l}\text { Hernández } \\
\text { Mariano JA et } \\
\text { al. } 2014^{29} \\
\text { México } \\
\text { SJR: Q3 }\end{array}$ & $\begin{array}{l}\text { Estudio } \\
\text { descriptivo } \\
\text { comparativo y } \\
\text { transversal }\end{array}$ & $64^{*}$ & SF-36 & $\begin{array}{l}\text { Comparar la } \\
\text { calidad de vida en } \\
\text { pacientes tratados } \\
\text { con DPCA y HD }\end{array}$ & $\begin{array}{l}\text { Los pacientes sometidos a DPCA } \\
\text { presentaron mejor calidad de vida. }\end{array}$ \\
\hline $\begin{array}{l}\text { Albañil Frías T } \\
\text { et al. } 2014^{30} \\
\text { España } \\
\text { SJR: Q3 }\end{array}$ & $\begin{array}{l}\text { Observacional } \\
\text { analítico } \\
\text { de corte } \\
\text { transversal }\end{array}$ & $43^{*}$ & $\begin{array}{l}\text { SF-36 } \\
\text { Karnofsky } \\
\text { Índice de } \\
\text { comorbilidad de } \\
\text { Charlson (ICC) } \\
\text { Variables } \\
\text { sociodemográficas }\end{array}$ & $\begin{array}{l}\text { Analizar la CVRS } \\
\text { de los pacientes } \\
\text { con IRC en HD } \\
\text { ambulatoria } \\
\text { y su relación } \\
\text { con el nivel de } \\
\text { dependencia. }\end{array}$ & $\begin{array}{l}\text { Los pacientes estudiados presentan } \\
\text { peor CVRS, afectando a todas las } \\
\text { dimensiones en comparación con los } \\
\text { valores de referencia de la población } \\
\text { general española. Las dimensiones } \\
\text { más afectadas respectivamente son } \\
\text { el rol físico, función social y función } \\
\text { física, siendo las menos afectadas } \\
\text { salud mental y rol físico. Esta peor } \\
\text { CVRS se asocia con mayor edad, } \\
\text { comorbilidad y dependencia. }\end{array}$ \\
\hline $\begin{array}{l}\text { Seok Hui Kang } \\
\text { et al. } 2017^{31} \\
\text { Korea } \\
\text { SJR: Q1 }\end{array}$ & Transversal & $1616^{*}$ & $\begin{array}{l}\text { Escalas } \\
\text { sociodemográficas } \\
\text { Fragilidad } \\
\text { Discapacidad } \\
\text { KDQ0L-SF } 1.3 \\
\text { SF-36 }\end{array}$ & $\begin{array}{l}\text { Evaluar las } \\
\text { diferencias en } \\
\text { la CVRS, la } \\
\text { fragilidad y la } \\
\text { discapacidad } \\
\text { según la } \\
\text { modalidad } \\
\text { de diálisis en } \\
\text { la población } \\
\text { coreana. }\end{array}$ & $\begin{array}{l}\text { No hubo diferencias significativas } \\
\text { en la fragilidad entre los pacientes } \\
\text { tratados con las dos modalidades de } \\
\text { diálisis; sin embargo, la discapacidad } \\
\text { era más común en pacientes con } \\
\text { EP que en pacientes con HD. EI } \\
\text { componente mental y el físico fueron } \\
\text { más favorables en los pacientes con } \\
\text { HD que en los pacientes con DP. Los } \\
\text { síntomas / problemas, el sueño, la } \\
\text { calidad de la interacción social y el } \\
\text { apoyo social fueron más favorables } \\
\text { en los pacientes con HD que en los } \\
\text { pacientes con DP; sin embargo, la } \\
\text { satisfacción del paciente y el estímulo } \\
\text { del personal de diálisis fueron más } \\
\text { favorables en los pacientes con DP } \\
\text { que en los pacientes con HD. }\end{array}$ \\
\hline $\begin{array}{l}\text { Iyasere OU et } \\
\text { al. } 2016^{32} \\
\text { Londres } \\
\text { SJR: Q1 }\end{array}$ & $\begin{array}{l}\text { Estudio } \\
\text { observacional } \\
\text { comparativo } \\
\text { de corte } \\
\text { transversal }\end{array}$ & $251^{*}$ & $\begin{array}{l}\text { SF-12 } \\
\text { RTSQ } \\
\text { HADS } \\
\text { Barthel Score }\end{array}$ & $\begin{array}{l}\text { Comparar la } \\
\text { CVRS y la función } \\
\text { física entre } \\
\text { pacientes de } \\
\text { mayor edad en DP } \\
\text { y HD. }\end{array}$ & $\begin{array}{l}\text { No hay diferencias en las medidas de } \\
\text { la calidad de vida y la función física } \\
\text { entre los pacientes mayores en DP } \\
\text { y los pacientes en HD, excepto por } \\
\text { la satisfacción con el tratamiento, } \\
\text { que es mayor en los pacientes en DP, } \\
\text { esta debe considerarse como una } \\
\text { alternativa a la HD para pacientes } \\
\text { mayores, permitiéndoles tomar sus } \\
\text { decisiones preferidas. }\end{array}$ \\
\hline
\end{tabular}




\begin{tabular}{|c|c|c|c|c|c|}
\hline $\begin{array}{l}\text { Autor, año y } \\
\text { país }\end{array}$ & $\begin{array}{l}\text { Diseño del } \\
\text { estudio }\end{array}$ & Muestra & $\begin{array}{l}\text { Instrumento de } \\
\text { medida }\end{array}$ & Objetivo & Conclusiones \\
\hline $\begin{array}{l}\text { Liebman S et } \\
\text { al. } 2016^{33} \\
\text { Noruega } \\
\text { SJR: Q1 }\end{array}$ & Retrospectivo & 1017 * & SF-36 & $\begin{array}{l}\text { Asociación de } \\
\text { los cambios en la } \\
\text { CVRS a lo largo } \\
\text { del tiempo con } \\
\text { la mortalidad en } \\
\text { un gran grupo de } \\
\text { pacientes de HD. }\end{array}$ & $\begin{array}{l}\text { Los médicos deben ser conscientes de } \\
\text { que muchos pacientes experimentan } \\
\text { un cambio significativo tanto en el } \\
\text { Componente Mental (CM) como en el } \\
\text { Físico (CF) en HD. Una disminución } \\
\text { en el CM de } \geq 5 \text { se asoció con un } \\
\text { aumento de la mortalidad. Se deben } \\
\text { evaluar las causas fundamentales y } \\
\text { buscar mitigar las disminuciones en } \\
\text { la calidad de vida siempre que sea } \\
\text { posible. }\end{array}$ \\
\hline $\begin{array}{l}\text { Carpena } \\
\text { Ramos EC et } \\
\text { al. } 2014^{34} \\
\text { Brasil } \\
\text { SJR: Q3 }\end{array}$ & Transversal & $317^{*}$ & SF-36 & $\begin{array}{l}\text { Comparar la } \\
\text { CVRS entre } \\
\text { los pacientes } \\
\text { con ERC en } \\
\text { tratamiento con } \\
\text { DP y HD. }\end{array}$ & $\begin{array}{l}\text { La CVRS es similar entre los } \\
\text { pacientes de HD y DP, excepto en } \\
\text { términos de dolor, que es menos } \\
\text { severo en los pacientes de DP. }\end{array}$ \\
\hline $\begin{array}{l}\text { Urzúa A et al. } \\
2011^{35} \\
\text { Chile } \\
\text { SJR: Q3 }\end{array}$ & $\begin{array}{l}\text { Revisión } \\
\text { sistemática }\end{array}$ & 128 ** & KDQ0L-SF 36 & $\begin{array}{l}\text { Analizar la } \\
\text { relación existente } \\
\text { entre las variables } \\
\text { edad, tiempo en } \\
\text { tratamiento, sexo, } \\
\text { apoyo familiar } \\
\text { percibido, salud } \\
\text { mental, estado de } \\
\text { salud percibido y } \\
\text { la CVRS en HD. }\end{array}$ & $\begin{array}{l}\text { La salud mental es un elemento } \\
\text { modulador de la CVRS en los } \\
\text { pacientes en HD, en tanto otros como } \\
\text { el sexo, el tiempo de diálisis o la edad } \\
\text { de los participantes no constituyeron } \\
\text { elementos relevantes en esta } \\
\text { evaluación. }\end{array}$ \\
\hline $\begin{array}{l}\text { Loon et al. } \\
2017^{36} \\
\text { Países Bajos } \\
\text { SJR: Q1 }\end{array}$ & $\begin{array}{l}\text { Ensayo } \\
\text { controlado } \\
\text { aleatorio } \\
\text { mulcicéntrico }\end{array}$ & $714^{*}$ & KDQOL-SF & $\begin{array}{l}\text { Comparar la } \\
\text { prevalencia de } \\
\text { deterioro según } \\
\text { KDQOL-SF entre } \\
\text { las diferentes } \\
\text { categorías de } \\
\text { edad y evaluar } \\
\text { la asociación de } \\
\text { estas subescalas } \\
\text { con la mortalidad } \\
\text { difiere entre } \\
\text { los pacientes } \\
\text { de diálisis } \\
\text { más jóvenes y } \\
\text { mayores. }\end{array}$ & $\begin{array}{l}\text { La edad avanzada se asocia con } \\
\text { niveles más bajos de funcionamiento } \\
\text { físico, mientras que el nivel de salud } \\
\text { emocional no se asocia con la edad. } \\
\text { El funcionamiento físico, la salud } \\
\text { emocional y el funcionamiento } \\
\text { social de los dominios KDQOL-SF se } \\
\text { asocian de forma independiente con } \\
\text { la mortalidad en pacientes de HD más } \\
\text { jóvenes y mayores prevalentes. }\end{array}$ \\
\hline $\begin{array}{l}\text { García Llana H } \\
\text { et al. } 2013^{37} \\
\text { España } \\
\text { SJR: Q2 }\end{array}$ & $\begin{array}{l}\text { Evaluación } \\
\text { mediante } \\
\text { autoinformes } \\
\text { e instrumentos } \\
\text { estandarizados }\end{array}$ & $61^{*}$ & $\begin{array}{l}\text { SF-36 } \\
\text { Escalas específicas }\end{array}$ & $\begin{array}{l}\text { Determinar la } \\
\text { relación entre } \\
\text { la adherencia, el } \\
\text { estado emocional } \\
\text { y la CVRS en } \\
\text { los pacientes } \\
\text { sometidos a } \\
\text { diálisis (HD y } \\
\text { DP). }\end{array}$ & $\begin{array}{l}\text { La modalidad de diálisis no aporta } \\
\text { diferencias en el estado emocional } \\
\text { o la adherencia específica a la } \\
\text { medicación, sin embargo, sí que está } \\
\text { relacionada con su adherencia general } \\
\text { al tratamiento y su CVRS. }\end{array}$ \\
\hline
\end{tabular}




\begin{tabular}{|c|c|c|c|c|c|}
\hline $\begin{array}{l}\text { Autor, año y } \\
\text { país }\end{array}$ & $\begin{array}{l}\text { Diseño del } \\
\text { estudio }\end{array}$ & Muestra & $\begin{array}{l}\text { Instrumento de } \\
\text { medida }\end{array}$ & Objetivo & Conclusiones \\
\hline $\begin{array}{l}\text { Barbero } \\
\text { Narbona E et } \\
\text { al. } 2016^{38} \\
\text { España } \\
\text { SJR: ND }\end{array}$ & $\begin{array}{l}\text { Estudio } \\
\text { descriptivo } \\
\text { de corte } \\
\text { transversal } \\
\text { prospectivo }\end{array}$ & $42^{*}$ & $\begin{array}{l}\text { Índice de Charlson } \\
\text { SF-12 } \\
\text { Índice de Barthel } \\
\text { Lawton-Brody } \\
\text { Yesavage }\end{array}$ & $\begin{array}{l}\text { Valorar y analizar } \\
\text { la percepción } \\
\text { de salud, la } \\
\text { capacidad } \\
\text { funcional, el } \\
\text { estado nutricional } \\
\text { y psicológico de } \\
\text { los pacientes de } \\
\text { HD y DP. }\end{array}$ & $\begin{array}{l}\text { Es importante conocer el efecto de } \\
\text { la enfermedad renal crónica y su } \\
\text { tratamiento sobre el paciente, para } \\
\text { así poder adecuar los cuidados de } \\
\text { enfermería. Los pacientes en DP tienen } \\
\text { sobrepeso y obesidad, comparados con } \\
\text { los de HD que mantienen cifras más } \\
\text { parecidas a la población general. La } \\
\text { autonomía es similar en ambos grupos, } \\
\text { en general, las personas perciben su } \\
\text { salud física peor que la emocional, } \\
\text { dándose esto en los pacientes de HD, lo } \\
\text { cual conlleva a un mayor sedentarismo. }\end{array}$ \\
\hline $\begin{array}{l}\text { Besey Ören et } \\
\text { al. } 2013^{39} \\
\text { Turquía } \\
\text { SJR: Q1 }\end{array}$ & Transversal & $300 *$ & $\begin{array}{l}\text { Escala de calidad de } \\
\text { vida }\end{array}$ & $\begin{array}{l}\text { Investigar los } \\
\text { factores que } \\
\text { afectan a la } \\
\text { calidad de vida de } \\
\text { los pacientes en } \\
\text { HD y DP, además } \\
\text { de proporcionar } \\
\text { una comparación } \\
\text { de la calidad de } \\
\text { vida entre ambos } \\
\text { grupos. }\end{array}$ & $\begin{array}{l}\text { Se concluyó que la calidad de vida se vio } \\
\text { afectada en los pacientes con HD y PD } \\
\text { y que el grupo de DP tenía una mejor } \\
\text { calidad de vida que el grupo de HD. Se } \\
\text { encontró que los valores de la calidad de } \\
\text { vida se vieron afectados por la edad, el } \\
\text { sexo, la educación, la situación laboral, } \\
\text { los ingresos, la duración de la diálisis, la } \\
\text { incidencia de peritonitis y enfermedades } \\
\text { adicionales. Las enfermeras que } \\
\text { trabajan en estas unidades podrían } \\
\text { minimizar los efectos negativos de } \\
\text { factores como la peritonitis y la anemia } \\
\text { en la calidad de vida y el pronóstico } \\
\text { mediante el uso de métodos de atención } \\
\text { más efectivos y la capacitación del } \\
\text { paciente. }\end{array}$ \\
\hline $\begin{array}{l}\text { Ruiz de Alegría } \\
\text { B et al. } 2013^{40} \\
\text { España } \\
\text { SJR: ND }\end{array}$ & $\begin{array}{l}\text { Estudio } \\
\text { longitudinal } \\
\text { de corte } \\
\text { transversal }\end{array}$ & $98^{*}$ & $\begin{array}{l}\text { MOS-SF36 } \\
\text { PNA } \\
\text { Formas de } \\
\text { Afrontamiento }\end{array}$ & $\begin{array}{l}\text { Conocer las } \\
\text { formas de } \\
\text { afrontamiento } \\
\text { más utilizadas } \\
\text { en primer año } \\
\text { de tratamiento } \\
\text { y analizar su } \\
\text { influencia en la } \\
\text { calidad de vida y } \\
\text { salud emocional. }\end{array}$ & $\begin{array}{l}\text { Las estrategias de evitación y } \\
\text { aproximación son factores que influyen } \\
\text { en el bienestar psicológico de los } \\
\text { pacientes en diálisis. Los pacientes que } \\
\text { utilizan estas estrategias de evitación } \\
\text { al inicio de diálisis están en riesgo de } \\
\text { empeorar su bienestar psicológico. } \\
\text { Es importante para los profesionales } \\
\text { identificar precozmente las estrategias } \\
\text { de evitación. }\end{array}$ \\
\hline $\begin{array}{l}\text { Nagasawa } H \text { et } \\
\text { al. } 2018^{41} \\
\text { Japón } \\
\text { SJR: Q1 }\end{array}$ & $\begin{array}{l}\text { Estudio } \\
\text { prospectivo } \\
\text { de corte } \\
\text { transversal }\end{array}$ & $84^{*}$ & $\begin{array}{l}\text { EQ-5D } \\
\text { KDQOL-SF } \\
\text { SF-36 }\end{array}$ & $\begin{array}{l}\text { Encuestar la } \\
\text { CVRS de los } \\
\text { pacientes en } \\
\text { diálisis y sus } \\
\text { cuidadores, para } \\
\text { analizar la posible } \\
\text { relación entre } \\
\text { ambos. }\end{array}$ & $\begin{array}{l}\text { Los cuidadores tendían a tener una } \\
\text { CVRS física más baja si sus pacientes } \\
\text { tenían una calidad de vida social } \\
\text { alta y una calidad de vida más baja } \\
\text { relacionada con la salud mental durante } \\
\text { la etapa temprana del tratamiento } \\
\text { de diálisis del paciente, y cuando } \\
\text { los pacientes experimentaban una } \\
\text { calidad de vida limitada orientada a la } \\
\text { enfermedad. }\end{array}$ \\
\hline $\begin{array}{l}\text { Atapour A et } \\
\text { al. } 2016^{42} \\
\text { Irán } \\
\text { SJR: Q3 }\end{array}$ & $\begin{array}{l}\text { Estudio } \\
\text { transversal }\end{array}$ & $92 *$ & $S F-36$ & $\begin{array}{l}\text { Comparar la } \\
\text { calidad de vida } \\
\text { de los pacientes } \\
\text { sometidos a TRS } \\
\text { entre HD o DP. }\end{array}$ & $\begin{array}{l}\text { No hubo diferencias significativas } \\
\text { entre las cualidades de la diálisis } \\
\text { en los dos grupos de pacientes. Los } \\
\text { aspectos de la calidad de vida, como el } \\
\text { funcionamiento físico, rol físico, el dolor } \\
\text { corporal, las percepciones generales } \\
\text { de salud y la puntuación general fueron } \\
\text { significativamente diferentes entre } \\
\text { los dos grupos. Si estos resultados se } \\
\text { fundamentan en estudios longitudinales } \\
\text { posteriores, la elección de diálisis podría } \\
\text { guiarse mejor en los pacientes por la } \\
\text { calidad de vida. }\end{array}$ \\
\hline
\end{tabular}




\begin{tabular}{|c|c|c|c|c|c|}
\hline $\begin{array}{l}\text { Autor, año y } \\
\text { país }\end{array}$ & $\begin{array}{l}\text { Diseño del } \\
\text { estudio }\end{array}$ & Muestra & $\begin{array}{l}\text { Instrumento de } \\
\text { medida }\end{array}$ & Objetivo & Conclusiones \\
\hline $\begin{array}{l}\text { Perales } \\
\text { Montilla CM } \\
\text { et al. } 2016^{43} \\
\text { España } \\
\text { SJR: ND }\end{array}$ & $\begin{array}{l}\text { Estudio } \\
\text { analítico } \\
\text { comparativo } \\
\text { de corte } \\
\text { transversal }\end{array}$ & $52^{*}$ & $\begin{array}{l}\text { SF-36 } \\
\text { ESS-R } \\
\text { HAD }\end{array}$ & $\begin{array}{l}\text { Comparar } \\
\text { la capacidad } \\
\text { predictiva de } \\
\text { los síntomas } \\
\text { somáticos y el } \\
\text { estado anímico } \\
\text { (depresión } \\
\text { y ansiedad) } \\
\text { sobre CVRS en } \\
\text { pacientes en HD. }\end{array}$ & $\begin{array}{l}\text { El estado anímico es un mejor } \\
\text { predictor de los componentes físico } \\
\text { y mental de la CVRS que el número } \\
\text { e intensidad de los síntomas físicos } \\
\text { informados por los pacientes. Se } \\
\text { demuestra la importancia de evaluar } \\
\text { e intervenir sobre los estados } \\
\text { emocionales negativos (depresión y } \\
\text { ansiedad) en los pacientes renales. } \\
\text { Estas intervenciones pueden } \\
\text { repercutir en una menor interferencia } \\
\text { de la enfermedad sobre la CVRS en el } \\
\text { paciente en hemodiálisis. }\end{array}$ \\
\hline $\begin{array}{l}\text { González FA et } \\
\text { al. } 2015^{44} \\
\text { Brasil } \\
\text { SJR: Q3 }\end{array}$ & $\begin{array}{l}\text { Estudio } \\
\text { transversal }\end{array}$ & $338^{*}$ & KDQOL-SF 36 & $\begin{array}{l}\text { Comparar la } \\
\text { CVRS de los } \\
\text { pacientes con } \\
\text { ERCA en estadio } \\
5 \text { que realizan HD } \\
\text { y DP domiciliaria. }\end{array}$ & $\begin{array}{l}\text { La DP fue más favorable con } \\
\text { respecto a la CVRS, debido a la gran } \\
\text { cantidad de elementos con resultados } \\
\text { significativos en comparación con la } \\
\text { HD. Sin embargo, las dos variables } \\
\text { de mayor importancia encontradas } \\
\text { en la HD (funcionamiento físico } \\
\text { y funcionamiento emocional) } \\
\text { terminaron teniendo un impacto } \\
\text { mucho mayor en el bienestar y la } \\
\text { vida diaria del paciente en el entorno } \\
\text { externo a la clínica que aquellos que } \\
\text { eran más altos en DP, haciendo de la } \\
\text { HD la más favorable para la calidad } \\
\text { de vida del paciente. }\end{array}$ \\
\hline $\begin{array}{l}\text { Gunalay S et } \\
\text { al. 2018 } \\
\text { Turquía } \\
\text { SJR: Q3 }\end{array}$ & $\begin{array}{l}\text { Transversal } \\
\text { descriptivo }\end{array}$ & $60 *$ & $\begin{array}{l}\text { MNA-SF } \\
\text { EQ-5D }\end{array}$ & $\begin{array}{l}\text { Determinar la } \\
\text { frecuencia de } \\
\text { desnutrición, la } \\
\text { calidad de vida y } \\
\text { la relación entre } \\
\text { ambas en ERCA. }\end{array}$ & $\begin{array}{l}\text { La CVRS puede mejorarse mediante } \\
\text { el diagnóstico temprano y el } \\
\text { tratamiento de la desnutrición en } \\
\text { pacientes en riesgo tanto en pacientes } \\
\text { en HD como en DP. }\end{array}$ \\
\hline $\begin{array}{l}\text { Karatas A et al. } \\
2018^{46} \\
\text { Turquía } \\
\text { SJR: Q3 }\end{array}$ & $\begin{array}{l}\text { Prospectivo } \\
\text { de corte } \\
\text { transversal }\end{array}$ & $240 *$ & $\begin{array}{l}\text { PSQI (Sueño) } \\
\text { BREF }\end{array}$ & $\begin{array}{l}\text { Planificar la } \\
\text { calidad del sueño } \\
\text { y la calidad de } \\
\text { vida en adultos } \\
\text { con ERCA. }\end{array}$ & $\begin{array}{l}\text { En conclusión, las enfermedades } \\
\text { crónicas deterioran gravemente } \\
\text { la calidad de vida y el sueño. En } \\
\text { el seguimiento de enfermedades } \\
\text { crónicas como la ERC, se deben } \\
\text { solicitar consultas sobre la calidad } \\
\text { de vida y la calidad del sueño, } \\
\text { especialmente para aumentar la } \\
\text { calidad del sueño. Los pacientes } \\
\text { deben ser remitidos a laboratorios } \\
\text { de sueño, dietistas, psicólogos, } \\
\text { psiquiatras y clínicas de medicina } \\
\text { complementaria cuando sea } \\
\text { necesario. }\end{array}$ \\
\hline
\end{tabular}

*: Tamaño de la muestra. **: Número de artículos revisado. SJR: Scimago Journal Ranking.

\section{Descripción de los resultados}

\section{Tipo de técnica de diálisis}

Del total de estudios incluidos en esta revisión, 15 de ellos estudiaron a pacientes exclusivamente con TRS de $\operatorname{HD}(41 \%)^{15,20,21,23-27,30,33,35,36,41,43,45}$, centrándose exclusivamente en DP tan solo un artículo ${ }^{22}$, siendo esta la modalidad menos estudiada en los últimos años y curiosamente, la técnica que aporta mayor autonomía e independencia al paciente, y mejor salud mental y física. 
En cambio, ambas técnicas (HD y DP) de forma conjunta se analizan en 14 estu$\operatorname{dios}^{17-19,28,29,31,32,34,37-40,42,44}$ representando un $38 \%$ del total.

Los pacientes cuya técnica de diálisis es la HD presentan peor CVRS que los pacientes que permanecen en DP19.28,36,39,43. El tratamiento de HD ocasiona mayor deterioro de la CVRS de los pacientes debido a las continuas sesiones a las que se tienen que someter, afectando a la independencia de la persona e incluso a su situación laboral y nivel socioeconómico, de aquellos que se encuentran en activo. A causa de esto se produce un deterioro tanto a nivel mental como en el componente físico $30,39,44,46$.

En cambio, en los pacientes en DP las puntuaciones para la CVRS suele ser más alta, debido principalmente a la mayor autonomía que aporta el tratamiento y una reducción de la dimensión dolor, puntuando más alta la satisfacción de los pacientes $28,31,32,36$.

\section{Instrumentos de evaluación empleados}

Tabla 3. Instrumentos genéricos más usados en España.

\begin{tabular}{|l|l|l|}
\hline INSTRUMENTO & $\begin{array}{l}\text { ÍTEMS/FORMA } \\
\text { DE ADMINISTRACIÓN }\end{array}$ & SUBESCALAS \\
\hline $\begin{array}{l}\text { Notthingham Health } \\
\text { Profile }\end{array}$ & $\begin{array}{l}38 \text { ítems } \\
\text { Autoadministrado }\end{array}$ & NO \\
\hline Sickness Impact Profile & $\begin{array}{l}136 \text { ítems } \\
\text { Autoheteroadministrado }\end{array}$ & N0 \\
\hline SF-36 Health Survey & $\begin{array}{l}36 \text { ítems } \\
\text { Autoheteroadministrado }\end{array}$ & SI (SF-12/ SF-8) \\
\hline EUROQ0L-5D & $\begin{array}{l}15 \text { ítems y } \\
\text { Escala Visual Analógica }\end{array}$ & N0 \\
\hline Láminas COOP-WONCA & $\begin{array}{l}\text { ítems } \\
\text { Autoheteroadministrado }\end{array}$ & N0 \\
\hline
\end{tabular}

Tabla 4. Instrumentos específicos más usados en España.

\begin{tabular}{l|l|l}
\hline INSTRUMENTO & $\begin{array}{l}\text { ITEMS/FORMA } \\
\text { DE ADMINISTRACIÓN }\end{array}$ & SUBESCALAS \\
\hline $\begin{array}{l}\text { Kidney Disease } \\
\text { Questionnarie }\end{array}$ & $\begin{array}{l}26 \\
\text { Heteroadministrado }\end{array}$ & NO \\
\hline $\begin{array}{l}\text { Kidney Transplant } \\
\text { Questionnarie }\end{array}$ & 25 & NO \\
\hline $\begin{array}{l}\text { Kidney Disease } \\
\text { and quality of life short form }\end{array}$ & $\begin{array}{l}\text { Autoadministrado } \\
\text { Autoadministrado }\end{array}$ & NO \\
\hline
\end{tabular}

Los estudios analizados recogen una gran variedad de instrumentos estandarizados para medir la calidad de vida, tanto de tipo genérico (mide la CVRS de forma general para enfermos de todo tipo de patologías) (Tabla 3) y de tipo específico (están centrados en un tipo de patología) (Tabla 4). Del total de estudios analizados, en $12(29 \%)^{18,21,26,29,30,32-34,37,38,42,43}$, se realiza el análisis de la CVRS con el cuestionario genérico SF-36 y/o SF12 (contienen misma temática pero son distintas versiones), utilizándose de forma exclusiva el cuestionario KDQOL-SF de tipo específico para enfermos renales en 14 estudios ${ }^{15,17,20,21,22-25,27,28,31,35,36,45}$, representando un $34,14 \%$ del total incluido en esta revisión. Solamente, en $3^{16,19,41}$ de ellos, se utiliza de forma conjunta los cuestionarios antes mencionados, para el análisis de la calidad de vida. A los instrumentos anteriormente descritos, en algunos casos, se le asocian factores sociodemográficos. En el resto de artículos, se utilizan escalas específicas para analizar otras dimensiones, destacando entre ellas escalas de ansiedad y depresión $22,26,43$.

\section{Diseño de los estudios}

Todos los estudios de investigación fueron de tipo observacional, de corte transversal en su mayo- ría $15,18,20-29,33,34,38,41,42,44-46$, siguiéndole un grupo variado de artículos $^{16,23,35,40,42}$, de tipo longitudinal, de tipo comparativo, etc, siendo por último 4 estudios de revisión sistemática $(12 \%)^{16,17,19,35}$.

\section{Factores sociodemográficos}

Edad: al tratarse de una enfermedad crónica, la edad se ve ligada al concepto de CVRS, de manera que existe una asociación entre esta variable y niveles bajos de CVRS, mientras más avanzada sea la edad peor CVRS perciben los propios pacientes $17,18,22,24,25,29,30,36,44,46$. Sin embargo, algunos autores documentan que la edad no tiene una influencia directa en la CVRS $35,38,43$.

Género: diferentes estudios encuentran que el sexo femenino tiene peor percepción de la CVRS que el masculino ${ }^{16,27,29,30,37,45}$, debido posiblemente, a una mayor percepción del dolor y carga social en su rutina diaria, por parte de las mujeres. En cambio, existen algunas publicaciones que recogen que el género no es una variable que influya en el análisis de CVRS $24,35,43$.

Estado Civil: no está muy establecido el papel de esta variable en la CVRS, sin embargo en varios artícu- 
$\operatorname{los}^{15,18,20,23,25,29,30,45,46}$ encuentran que estar casado o vivir en pareja tiene resultados positivos para la calidad de vida, mostrando peor calidad de vida, aquellas personas que no tienen un núcleo familiar y tienen menor apoyo familiar.

Nivel de Estudios: aunque no de forma muy determinante, existen estudios, en los que se recoge que un nivel de estudios primarios o bajo está relacionado con una menor CVRS 22,25,27,36; existiendo otros estudios en los que se relaciona un elevado nivel de estudios con una mejor puntuación en la CVRS 27,46 .

Situación Laboral y Nivel Socioeconómico: debido a la avanzada edad de los pacientes y a la cronicidad de la enfermedad, la situación laboral de la población, en su mayoría, es no activa, predominando los jubilados. Pero sí que existen algunos estudios, en los que se relaciona la situación laboral de los enfermos con ERCA y terapia sustitutiva renal (TSR) con la CVRS, en los que se refleja que ambas situaciones son factores predisponentes de incapacidad laboral para la población en activo, lo que conlleva a situaciones de estrés y precariedad para los cuidados, viéndose esto reflejado en una menor CVRS $15,30,37,38,46$. Además en 2 artículos, se relaciona un nivel socioeconómico alto con mejor percepción de la CVRS en pacientes con TRS 44,46 .

\section{Comorbilidad asociada}

La comorbilidad asociada a los pacientes en tratamiento de diálisis aparece en los estudios analizados como una variable altamente influyente sobre la CVRS, asociándose una mayor comorbilidad con una peor CVRS en los pacientes en diálisis ${ }^{15,16,18,20,23,22-24,30-32,38,44,45}$; siendo frecuente la presencia de hipertensión arterial y diabetes mellitus de forma conjunta, 0 aislada.

\section{Ansiedad y depresión}

Una de las variables más influyente en la percepción de la calidad de vida es padecer ansiedad y/o depresión. Diferentes autores destacan la asociación entre esta variable y la CVRS: Los pacientes sometidos a diálisis suelen presentar una alta prevalencia de ansiedad y/o, depresión, y esta repercute negativamente en la CVRS, tanto en el componente físico como mental. Esta asociación es producida por el grado de afectación que produce en el componente físico, de manera que limita a los pacientes a realizar las actividades básicas de la vida diaria (ABVD) como de costumbre, presentando las mujeres de mayor edad una tendencia a padecer depresión moderada, y los hombres depresión leve $15-17,20,21,22,25,26,37,43,44$.

\section{Dolor}

El dolor es una variable que influye de forma negativa en la CVRS de los pacientes en diálisis, siendo este menos frecuente en aquellos sometidos a tratamiento de DP. EI dolor más frecuente es de origen musculo-esquelético y calambres en miembros inferiores, teniendo esto una repercusión directa en las actividades básicas de la vida diaria, reduciendo su autonomía e independencia, y por consiguiente reduciendo su calidad de vida, siendo además, más frecuente en las mujeres ${ }^{18,23,27,34,37,46}$.

\section{Grado de dependencia}

Debido a la cronicidad de la ERCA y a las limitaciones físicas que esta ocasiona, existen algunos estudios en los que se relaciona el grado de dependencia de los pacientes con peor puntuación en la CVRS, sobre todo en las dimensiones físicas ${ }^{27,30,41,43}$.

\section{Discusión}

Con esta revisión se pretendía conocer la bibliografía existente sobre la CVRS de los pacientes con ERCA en diálisis, en cualquiera de sus modalidades, así como las variables más influyentes que intervienen en la misma. La disminución de la CVRS está presente en la mayoría de los pacientes de diálisis, en comparación con la población sana de referencia ${ }^{16,17,35}$. En la ERCA conforme va avanzando y progresando a estadios finales, se va produciendo un deterioro de todas las dimensiones vitales de la persona que la padece, afectando de forma importante a su vida, dándose por consiguiente una reducción en la calidad de vida de estos enfermos. Esto no solo ocurre debido al avance de la enfermedad y los síntomas, sino que también se debe a las características de las TRS a los que tienen que someterse, provocando esto cambios, por ejemplo en su situación laboral o en la dieta ${ }^{4}$.

La mayoría de los estudios revisados sobre la CVRS en diálisis, se centran en el estudio de la misma en HD, siendo escasos los artículos que analizan esta variable en DP, en exclusiva22; a pesar de que esta técnica proporciona mayor autonomía e independencia al paciente, aunque no sea un procedimiento viable para todos los pacientes que precisen una TRS, por no cumplir los requisitos necesarios, o por ser poco conocida entre ellos ${ }^{47,48}$. En cambio, ambas técnicas (HD y DP) se analizan de forma 
conjunta en bastantes estudios. En cualquier caso, con ambas técnicas se tiene peor percepción de CVRS en general. Al comparar ambos procedimientos, los pacientes que se encuentran en HD, presentan peores puntuaciones en la mayoría de las dimensiones de la CVRS que se analizan (componente físico y mental, rol emocional, salud general, bajas posibilidades de poder trabajar). La mejor puntuación de los pacientes en DP puede deberse, a que esta técnica proporciona más autonomía al paciente por no tener que acudir al centro del tratamiento, y suele haber un sesgo de selección por parte de los pacientes al elegir la técnica²2.

Respecto a los instrumentos de evaluación empleados para medir la CVRS, se puede afirmar que el más utilizado es el de tipo mixto KDQOL-SF para los enfermos renales, ya que KDQOL fue diseñado para los pacientes sometidos a diálisis (HD y DP) e incluye el SF-36 como núcleo genérico, de tal manera que contiene los 43 ítems del específico y los 36 ítems del genérico, pudiendo evaluarse en esta versión la salud general en uno de los ítems ${ }^{49}$.

Como se ha podido observar en la Tabla 2, el 30\% de artículos utilizados en esta revisión son revisiones sistemáticas y el $70 \%$ de los estudios analizados son de tipo observacional. Como es lógico en la evaluación de esta variable, no se han encontrado estudios de tipo experimental.

En cuanto a los factores sociodemográficos que se analizan en los estudios incluidos en esta revisión, se destaca que tanto una edad avanzada como el sexo femenino son dos variables que influyen en peor percepción de la CVRS de los pacientes en diálisis, debido posiblemente a la cronicidad de la enfermedad y a una mayor carga social de la mujer en su día a día. Otra de las variables influyentes de forma negativa, es la situación laboral y nivel socioeconómico, especialmente en los pacientes en HD, debido a la dificultad de compatibilizar las sesiones de diálisis con un puesto de trabajo ${ }^{50}$. Además de estas, existen otras variables que también influyen de forma negativa en la CVRS en los pacientes en diálisis, aunque de forma menos relevante, como son el nivel de estudios, donde hay artículos en los que se recoge que pacientes con estudios bajos o primarios se asocia a una menor CVRS, al igual que el estado civil, reflejándose una mayor CVRS en aquellos pacientes que viven en pareja o están casados $^{51}$.

La comorbilidad asociada de los pacientes en diálisis es una variable asociada a peor CVRS, debido a que entre otras, depende de la edad avanzada de los pacientes y cronicidad de la enfermedad renal, siendo las dos patologías asociadas más frecuentes la diabetes mellitus y la hipertensión arterial. Por ello, prevenir la aparición de otras patologías asociadas a la enfermedad renal, permite tratar las complicaciones que puedan presentarse, mejorar la supervivencia y propiciar una mejora en la percepción que el paciente tiene sobre su estado de salud ${ }^{30}$.

Dado que la CVRS es un concepto multidimensional mediante el cual se evalúa al paciente de forma integral, en la persona con afectación renal, se debe tener en cuenta no solo los síntomas o alteraciones físicas, sino también el estado psicológico. Entre las alteraciones psicológicas que suelen estar presentes, se pueden destacar la influencia de ansiedad y depresión en la CVRS, ya que la presencia de estas alteraciones juntas o por separado influye de manera significativa en peor percepción de la CVRS, ocurriendo en su mayoría por la afectación del componente físico y de forma moderada en el sexo femenino $0^{50,51}$.

Por último, destacar la asociación encontrada entre el dolor y grado de dependencia con la disminución de la CVRS en los pacientes con ERCA; debido posiblemente, a la repercusión que el dolor tiene en la realización de las actividades básicas de la vida diaria y ciertas limitaciones, sobre todo físicas, asociadas a una edad avanzada de estos pacientes ${ }^{30}$.

\section{Limitaciones del estudio}

Las principales limitaciones de esta revisión han sido las propias de los estudios observacionales, la falta de homogeneidad de los estudios, pues los resultados son muy dependientes del contexto cultural en el que han sido llevados a cabo; por lo que algunos resultados no son nada concluyentes. Por otra parte la población analizada en los estudios de CVRS de pacientes en HD es muy superior a la analizada en estudios en DP, por lo que la comparación entre ambos procedimientos no es equitativa.

\section{Consideraciones prácticas}

Teniendo en cuenta que la mayoría de los pacientes en diálisis presentan una mala CVRS, sería fundamental la actuación por parte de enfermería, identificando los factores más influyentes en esta realidad e interviniendo para proporcionar al paciente un mejor estado de bienestar y a ser posible, mejor calidad de vida. 
Según los resultados de la presente revisión podemos concluir que los pacientes con ERCA en tratamiento con diálisis tienen disminuida la CVRS en todas las dimensiones de esta. Los pacientes en HD presentan peor CVRS que los pacientes en DP, aunque existe un sesgo de selección en los estudios.

Las variables más influyentes en la afectación de la CVRS en los pacientes en diálisis, son la edad avanzada, el sexo femenino, comorbilidad asociada, dolor con limitaciones, presencia de ansiedad y/o depresión y mayor grado de dependencia.

Los instrumentos utilizados para medir la CVRS en los pacientes con ERCA son tanto de tipo genérico, como el SF-36 y/o SF-12, como de tipo específico, como el KDQOL-SF para enfermos renales, siendo este último el más utilizado en la bibliografía.

Teniendo en cuenta la influencia que ejerce la ERCA en la CVRS, sería conveniente que esta se midiera de forma estandarizada, al inicio del tratamiento sustitutivo, de la misma forma que otras variables biológicas, para poder diseñar estrategias y/o intervenciones y así poder prevenir complicaciones y retrasar la afectación en la calidad de vida que esta enfermedad condiciona.

Recibido: 16-05-19

Revisado: 20-05-19

Modificado: 22-05-19

Aceptado: 29-05-19

\section{Bibliografía}

1. Sociedad Española de Nefrología. La Enfermedad Renal Crónica en España 2018. SEN [Edición Electrónica]. 2018;1-21. [Consultado 14 de marzo de 2019]. Disponible en: http://www.senefro.org/contents/webstructure/comunicacion/SEN_dossier_Enfermedad_Renal_Cro.pdf.

2. De Francisco ALM, Otero $A$. Insuficiencia renal oculta: Estudio EPIRCE. Nefrologia. 2005;25(Supl 4):66-71.
3. National Kidney Foundation. KDOQI Clinical Practice Guidlines and Clinical Practice Recommendations for 2006 Updates: Hemodialysis Adequacy, Peritoneal Dialysis Adequacy, Vascular Access. Am J Kidney Dis [Edición Electrónica]. 2006;48:1322. (Consultado 14 de marzo de 2019). Disponible en: http://www.ncbi.nlm.nih.gov/pubmed/1704 4433.

4. Pabón-Varela $Y$, Saray Páez-Hernández K, Dayanna Rodríguez-Daza K, Eustralia Medina-Atencia C, López-Tavera M, Salcedo-Quintero LV, et al. Revista de la Facultad de Ciencias de la Salud Artículo de Revisión Calidad de vida del adulto con insuficiencia renal crónica, una mirada bibliográfica adult's life quality with chronic kidney disease, a bibliographic view resumen. Insufic Renal; Adulto. 2015;12(2):157-63.

5. Botero de Mejía BE, Pico Merchán ME. Calidad de vida relacionada con la salud (CVRS) en adultos mayores de 60 años. Hacia la Promoción la Salud [Edición Electrónica]. 2007;12:11-24. (Consultado 14 de marzo de 2019). Disponible en: http://www. scielo.org.co/pdf/hpsal/v12n1/v12nla01.pdf.

6. Soto M, Failde I. La calidad de vida relacionada con la salud como medida de resultados en pacientes con cardiopatía isquémica. Rev la Soc Esp del Dolor. 2004;11(8):505-14.

7. Tuesca R. La calidad de vida, su importancia y cómo medirla. Salud Uninorte. 2005;21:76-86. (Consultado 14 de marzo de 2019). Disponible en: http:// rcientificas.uninorte.edu.co/index.php/salud/article/ viewArticle/4113.

8. Velarde $E, M c ~ J$, Carlos ), Mc A-F. Consideraciones metodológicas para evaluar la calidad de vida Velarde-Jurado E, Avila-Figueroa C. Methods for quality of life assessment. Salud Publica Mex [Internet]. 2002; 44(5):448-63.

9. Ruiz MA, Pardo A. Calidad de vida relacionada con la salud: definición y utilización en la práctica médica. PharmacoEconomics Spanish Res Artic. 2013;2(1):31-43.

10. Férnandez R, Martín A, Barbas MJ, González MJ, Alonso MI, Ortega JM. Accesos vasculares y calidad de vida en la enfermedad crónica renal terminal. Angiologia. 2005;57(Supl 2):185-98. 
11. Ferrer G, Rajmil M, Rebollo L, Permanyer-Miralda P, Quintana G, Santed JM, et al. El cuestionario de salud SF-36 español: una década de experiencia y nuevos desarrollos. Gac Sanit. 2005;19(2):135-50.

12. Alonso J. Descripción del KDQOL - SF. Grup Investig en Serv Sanit Dr Aigüader, Barcelona, Esp. 2009;88:4. (Consultado 16 de marzo de 2019). Disponible en: https://www.rand.org/content/dam/ rand/.../kdqol/KDQ0L-36\%20U S\%20Spanish. pdf.

13. Hutton B, Catalá-López F, Moher D. La extensión de la declaración PRISMA para revisiones sistemáticas que incorporan metaanálisis en red: PRISMA-NMA. Med Clin (Barc). 2016; (xx):10-4.

14. Vandenbroucke JP, Elm E Von, Altman DG, Gøtzsche $P C$, Mulrow CD, Pocock SJ, et al. Explicación y elaboración de estudios observacionales en epidemiología Mejorar la comunicación y elaboración (STROBE): explicación. 2009;23(2):1-28.

15. Perez C, Riquelme G, Scharage J, Armijo I. Relación entre calidad de vida y representación de enfermedad en personas con enfermedad renal crónica terminal en tratamiento con hemodiálisis. Enferm Nefrol. 2015;18(2):89-96.

16. Rebollo-Rubio A, Morales-Asencio JM, Pons-Raventos ME M-FJ. Review of studies on health related quality of life in patients with advanced chronic kidney disease in Spain. Nefrologia. 2015;35(1):92109.

17. Zazzeroni L, Pasquinelli G, Nanni E, Cremonini V, Rubbi I. Comparison of Quality of Life in Patients Undergoing Hemodialysis and Peritoneal Dialysis: A Systematic Review and Meta-Analysis. Kidney Blood Press Res. 2017;42(4):717-27.

18. Seguí $A$, Amador $P$, Peris $A$, Ramos A. Calidad de vida en pacientes con insuficiencia renal crónica en tratamiento con diálisis. Rev Soc Esp Enferm Nefrol. 2010;13(3):155-60.

19. Boateng EA, East L. The impact of dialysis modality on quality of life: a systematic review. Journal of Renal Care. 2011;37(4):190-200.

20. Zúñiga C, Dapueto J, Muller H, Kirsten L, Alid R, Ortiz L. Evaluación de la calidad de vida en pacien- tes en hemodiálisis crónica mediante el cuestionario "Kidney Disease Quality of Life (KDQOL-36)". Revista Médica Chile. 2009;137:200-7.

21. Perales CM, García A, Reyes del Paso GA. Predictores psicosociales de la calidad de vida en pacientes con insuficiencia renal crónica en tratamiento de hemodiálisis. Nefrología. 2012;32(5):622-30.

22. Varela L, Vázquez MI, Bolaños L, Alonso R. Predictores psicológicos de la calidad de vida relacionada con la salud en pacientes en tratamiento de diálisis peritoneal. Nefrología. 2011;31(1):97-106.

23. Cuevas MA, Saucedo RP, Romero G, García JA, Hernández A. Relación entre las complicaciones y la calidad de vida del paciente en hemodiálisis. Enferm Nefrol. 2017;20(2):112-19.

24. Ho SE, Ho CC, Norshazwani $\mathrm{N}$, Teoh KH, Ismail HS, Jaafar MZ, Das S. Perception of quality of life amongst end stage renal failure patients undergoing haemodialysis. SEU Roma. 2013;164(4). [Consultado 14 marzo 2019]. Disponible en: http://www. seu-roma.it/riviste/clinica_terapeutica/apps/autos. php?id=1231.

25. Barros L, Herazo Y, Aroca G. Calidad de vida relacionada con la salud en pacientes con enfermedad renal crónica. Revista Facultad de Medicina. 2015;63(4):641-47. Disponible en: https:// revistas.unal.edu.co/index.php/revfacmed/article/ view/49805/54037.

26. Millán R, Gómez C, Gil F, Oviedo G, Villegas F. Calidad de vida relacionada con la salud y prevalencia de síntomas depresivos y ansiosos en pacientes en hemodiálisis, en 6 unidades renales de Bogotá, Colombia. Revista Colombiana Psiquiatría. 2009:38(4). [Consultado 16 marzo 2019]. Disponible en: https: //www.redalyc.org/html/806/80615450004/.

27. Adell M, Salvado T, Casado L, Andújar J, Ramiro M, Mencía A. Valoración de la calidad de vida y técnicas de diálisis mediante un cuestionario de resultados percibidos por el paciente. Enferm Nefrol. 2015;18(1):101-37.

28. López y López LR, Baca $A$, Guzmán $P M$, Ángeles $A$, Ramírez del Pilar R, López DS et al. Calidad de vida en hemodiálisis y diálisis peritoneal tras cuatro años de tratamiento. Med Int Méx. 2017;33(2):177- 
84. [Consultado 14 marzo 2019]. Disponible en: http://www.scielo.org.mx/scielo.php?script=sci_arttext\&pid=S018648662017000200177.

29. Hernández JA, Castañeda AC, Burciaga CY, Garvalena MJ. Calidad de vida en pacientes con tratamiento sustitutivo renal: diálisis peritoneal continua ambulatoria y hemodiálisis. Revista Iberoamericana de eduación e investigación en enfermería. 2014;4(1):67-74.

30. Albañil T, Ramírez MC, Crespo R. Análisis de la calidad de vida en pacientes en hemodiálisis ambulatoria y su relación con el nivel de dependencia. Enferm Nefrol. 2014;17(3):167-74.

3. Seok K, Young J, Young S, Chull J. Effect of dialysis on frailty phenotype, disability and health-related quality of life in maintenance dialysis patients. Plos One. 2017; 12(5):e0176814.

32. Iyasere $0 U$, Brown $E A$, Johansson $L$, Huson $L$, Smee J, Maxwell A, et al. Quality of life and physical function in older patients on dialysis: A comparison of assisted peritoneal dialysis with hemodialysis. American Society of Nephrology. 2016;11(3):42330.

33. Liebman S, Li NC, Lacson E. Change in quality of life and one-year mortality risk in maintenance dialysis patients. Qual Life Res. 2016; 28(9):2295-306.

34. Carpena EC, Santos IS, Vargas R, Grahl JM. Quality of life of chronic renal patients in peritoneal dialysis and hemodialysis. J Bras Nefrol. 2015; 37(3):297-305.

35. Urzúa A, Pavlov R, Cortés R, Pino V. Factores psicosociales relacionados con la calidad de vida en salud en pacientes hemodializados. Terapia Psicológica. 2011; 29(1):135-40.

36. Loon IN, Bots ML, Boereboom FTJ, Grooteman MPC, Blankestijn PJ,Dorpel MA et al. Quality of life as indicator of poor outcome in hemodialysis: relation with mortality in different age groups. BMC Nephrology. 2017;18:217.

37. García H, Remor E, Selgas R. Adherence to treatment, emotional stage and quality of life in patients with end-stage renal disease undergoing dialysis. Psicothema. 2013;25(1):79-86.
38. Barbero E, Tejeda E, Herrera C, García SM, Gascó $\mathrm{N}$, Junyent $\mathrm{E}$. Estudio comparativo del estado físico, mental y percepción de calidad de vida relacionada con la salud de los pacientes en diálisis. Enferm Nefrol. 2016;19(1):29-35.

39. Besey 0, Nuray E. Quality of life in chronic hemodialysis and peritoneal dialysis patients in Turkey and related factors. Int Journal of Nursing Practise. 2013;19(6):547-56.

40. Ruíz de Alegría B, Basabe N, Saracho R. El afrontamiento como predictor de la calidad de vida en diálisis: un estudio longitudinal y multicéntrico. Nefrología. 2013; 33(3):342-54.

41. Nagasawa H, Sugita I, Tachi T, Esaki H, Yoshida A, Kanematsu $Y$, et al. The relationship between dialysis patients'quality of life and caregivers' quality of life. Front Pharmacol. 2018;9:770.

42. Atapour A, Nasr S, Momeni A, Taheri D, Dolatkhah $S$. A comparison of the quality of life of the patients undergoing hemodialysis versus peritoneal dialysis and its correlation to the quality of dialysis. Saudi J Kidney Dis Transpl. 2016;27(2):270-80.

43. Perales CM, Duschek $S$, Reyes del Paso GA. Calidad de vida relacionada con la salud en la enfermedad renal crónica: relevancia predictiva del estado de ánimo y la sintomatología somática. Nefrología. 2016;36(3):275-82.

44. Gonzáles F, Fernández I, Camargo JM, Bucaneve J, Prado NM, Terumy $C$ et al. Quality of life in chronic renal patients on hemodialysis or peritoneal dialysis: a comparative study in a referral service of $\mathrm{Cu}$ ritiba-PR. J Bras Nefrol. 2014;37(4):467-74.

45. Gunalay S, Kilic $Y$, Akar H, Mergen $H$. The relationship between malnutrition and quality of life in hemodialysis and peritoneal dialysis patients. Rev Assoc Med Bras. 2018;64(9):845-52.

46. Karatas A, Canakci E, Turkmen E. Comparison of sleep quality and quality of life indexes with sociodemographic characteristics in patients with chronic kidney disease. Niger J Clin Pract. 2018;21(1): 1461-7.

47. Moreiras Plaza M. De dónde venimos y a dónde vamos en diálisis peritoneal: identificando barreras y estrategias de futuro. Nefrología. 2014;34(6):756-67. 
48. Rodríguez A, Pérez M. Avances recientes y perspectivas futuras en diálisis peritoneal. XXIX SEDYT 2007;28(4):158-64. [Consultado 14 marzo 2019]. Disponible en: https://www.elsevier.es/es-revistadialisis-trasplante-275-articulo-avancesrecientes-perspectivas-futuras-dialisis-13113115.

49. Chaves K, Duarte A, Vesga J. Adaptación transcultural del cuestionario KDQOLSF 36 para evaluar calidad de vida en pacientes con enfermedad renal crónica en Colombia. Revista Med. 2013;21(2):34-42.

50. Guerra V, Sanhueza 0, Cáceres M. Calidad de vida de personas en hemodiálisis crónica: relación con variables sociodemográficas, médico-clínicas y de laboratorio. Rev Latino-Am Enfermagem. 2012; 25(5):10 pantallas. [Consultado 16 marzo 2019]. Disponible en: http://www.scielo.br/scielo.php?pi$\mathrm{d}=$ S010411692012000500004\&script=sci_arttext\&tIng=es.

51. Pérez C, Dosis A, Díaz L, Villavicenso P. Efectos de la hemodiálisis en la calidad de vida en los usuarios. Horiz Enferm. 2009;20(1):57-65.

Este artículo se distribuye bajo una Licencia Creative Commons Atribución-NoComercial 4.0 Internacional. https://creativecommons.org/licenses/by-nc/4.0/

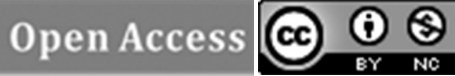

